\title{
Novel Band-Reject Microwave Filter by C-Shaped DGS with Improved $Q$ Factor
}

\author{
Mostafa Attaran Kakhki and Mohammad H. Neshati
}

\begin{abstract}
In this paper a novel $C$-shaped Defected Ground Structure (DGS) on a microstrip line is proposed to provide a frequency rejection property with an improved $Q$ factor at 2.4 GHz. Compared to the other DGS introduced structures in literature, the new structure has a simple and compact shape and its rejection characteristic is steeper at the stop band than the other reported structures. Different parameters of the DGS have been optimized for the best frequency response to reject the centre frequency. The proposed DGS is successfully designed and fabricated and the measured results are in a very good agreement with the simulated results.
\end{abstract}

Index Terms-Defected ground structure (DGS), patterned ground structures, bandreject filter, $\mathbf{Q}$ factor.

\section{INTRODUCTION}

Recently, using microstrip lines with defected ground structures (DGSs) have offered many applications at microwave frequencies as a filter due to compact size and better frequency characteristics. Also, at the design frequency of operation, one can achieve desirable features using these novel structures. Moreover, For the purpose of integrating with active and passive device, various research results have dealt with DGS [1]. The DGS structures provide a few resonance properties in transfer characteristics such as $R L C$ circuits. Corresponding to the shape and size of defect pattern, parallel or series resonance is introduced which can be used in microwave band reject filters. Using DGS, the effective inductance of the microstrip line could be increased lead to a longer electrical length of transmission line than that of a conventional line, and so compact microwave circuits can be implemented [2].

Photonic band gap (PBG) structures have a periodic configuration and provide rejection at certain frequencies. Too many design parameters such as number of lattice, lattice spacing, lattice shapes, and relative volume fraction effect on the bandgap properties. Hence, it is difficult to find the PBG structure's equivalent circuit and parameters; and also it is difficult to use a PBG circuit for the design of the microwave or millimeter-wave components [3]. On the other hand, the DGS structures can be modeled by simple resonant circuits and their parameter extraction is simple and they can be useful in the design of microwave and millimeter-wave circuits [4]. However, the modeling procedure for a DGS should be preceded to apply appropriately special characteristics of a DGS to a practical circuit. Finding the equivalent circuit and parameters of a DGS, various efforts

Manuscript received October 14, 2012; revised November 25, 2012.

Mostafa Attaran Kakhki is with Electrical Department of Ferdowsi University of Mashhad, Mashhad, Iran (e-mail: m.attarankakhki@ieee.org). are required [5].

In microwave applications, the band rejection property and the slow-wave effect of the DGS are used in the design of divider, filter, and amplifier circuits [6-11]. It is necessary to use a high $Q$-factor filter to suppress an unwanted signal, which is closely located from the desired signal in the spectrum. The dumbbell or spiral shapes have been used as a conventional defect of DGS. But, for narrowband rejection applications, their frequency characteristics are not adequate. The spiral DGS provides steeper rejection property than the dumbbell DGS, but the $Q$ factor of the spiral DGS is usually smaller than 10 .

In this paper, a new $C$-shaped DGS is proposed to provide a high $Q$ - factor at $2.4 \mathrm{GHz}$ as a band reject filter. A parametric study is carried out and the effects of DGS parameters are numerically investigated on the frequency response of the filter using HFSS. The proposed DGS filter provides $Q$ - factor of 57, which is a higher than the $Q$-factor of the conventional DGSs. The proposed DGS structure is shown in Fig. 1. The line width is chosen for the microstrip line impedance of $50 \Omega$. RF-35 PC-board with thickness of 60 -mil $(1.524 \mathrm{~mm})$ and dielectric constant of 3.5 is used in our study. The dimensions of the $C$-shaped, as labeled in Fig. 1 -b are $A=D=G=0.2 \mathrm{~mm}, B=1 \mathrm{~mm}, H=3 \mathrm{~mm}, C=14.2 \mathrm{~mm}$, and PC-boars size is set to $30 \times 40 \mathrm{~mm}^{2}$.

\section{Simulation Results of The Proposed DGS}

The DGS structure is simulated using HFSS. The numerical results of return loss, $S_{11}$ and forward transmission $S_{12}$ are shown in Fig. 2 versus frequency for two ranges. Fig 2-a shows that two parallel resonance frequencies around 2 $\mathrm{GHz}$ and $8 \mathrm{GHz}$ are provided by the proposed filter. Moreover, from Fig 2-b, it can be seen that at the exact lower resonance frequency is at $2.42 \mathrm{GHz}$, the signal attenuation is $40 \mathrm{~dB}$. The rejection bandwidth is $20 \mathrm{MHz}$ and $\mathrm{Q}$-factor is 121.

\section{PARAMETRIC STUDY}

A parametric study is investigated and the effects of DGS parameters on the characteristics of the DGS filter are carried out. First, the effect of $D$ is on the frequency response of the filter. The simulated results are shown in Fig. 3, which can b e seen that increasing $D$, from $0.2 \mathrm{~mm}$ to $0.4 \mathrm{~mm}$, resonance frequency and rejection bandwidth are increased, while other parameters are fixed to $B=1 \mathrm{~mm}, A=G=0.2 \mathrm{~mm}, H=3 \mathrm{~mm}$ and $C=14.2 \mathrm{~mm}$. Also, the obtained results show that $D$ has a weak effect on $Q$-factor. 


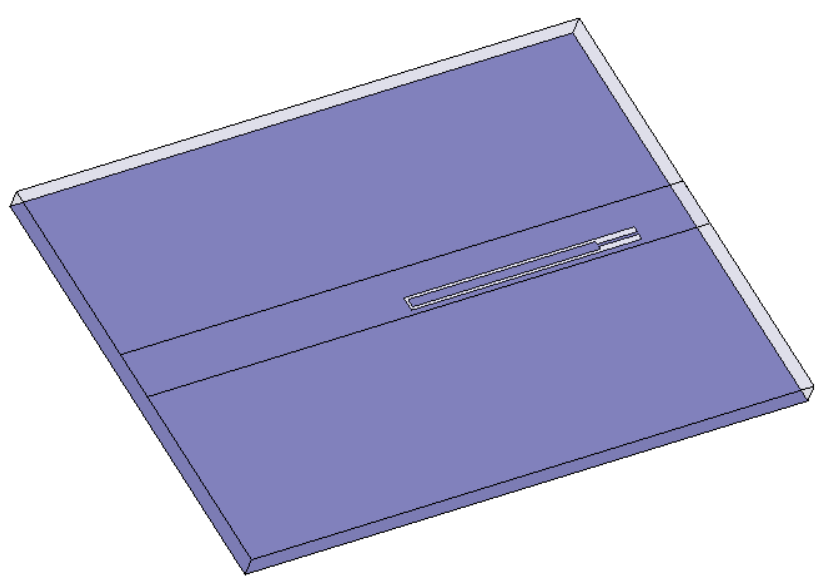

(a)

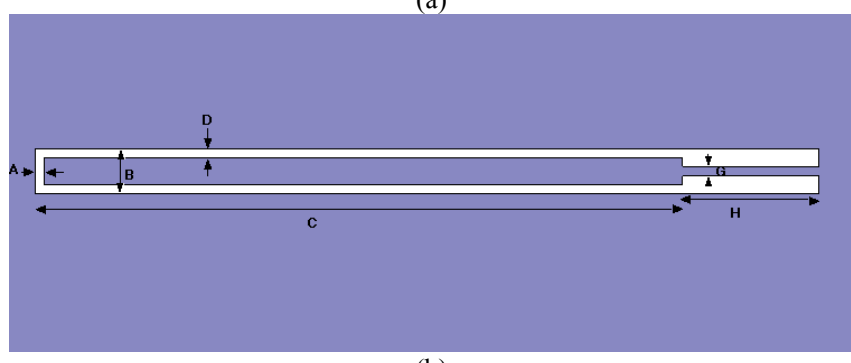

(b)

Fig. 1. Geometry of the $C$-shaped DGS filter: a) 3-D view, b) bottom view.

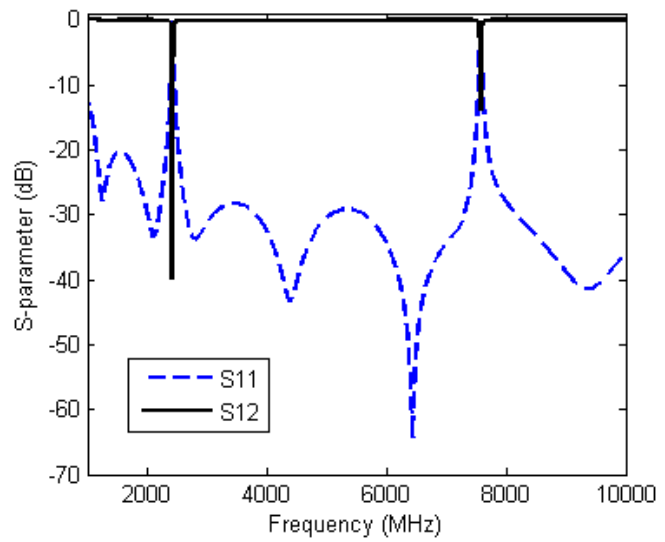

(a)

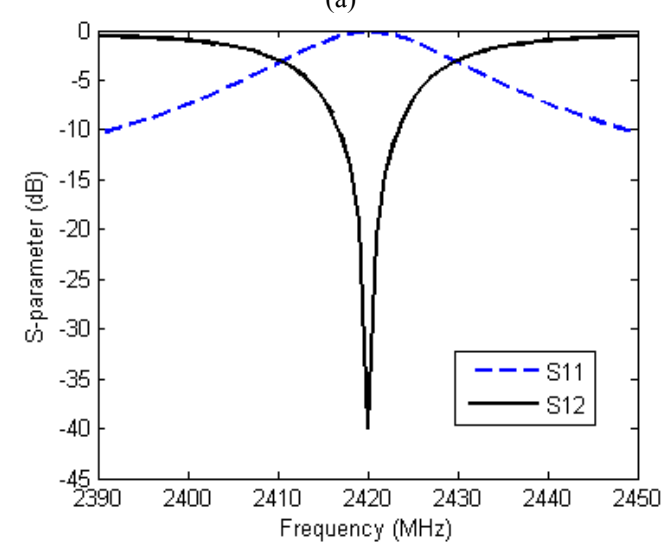

(b)

Fig. 2. Simulated $S_{12}$ of the DGS filter: a) $1 \mathrm{GHz}$ to $10 \mathrm{GHz}$, b) $2.39 \mathrm{GHz}$ to $2.45 \mathrm{GHz}$.

Fig. 4 shows the simulated $S_{12}$ of the proposed DGS filter versus $C$, varying from $12.2 \mathrm{~mm}$ to $15.2 \mathrm{~mm}$ with $1 \mathrm{~mm}$ steps, while other parameters are $B=1 \mathrm{~mm}, A=D=G=0.2 \mathrm{~mm}$ and $H=3 \mathrm{~mm}$. The simulated results clearly indicate that by increasing $C$, resonance frequency is decreases and rejection bandwidth variation is small. Hence, $Q$ - factor is increased.

Fig. 5 shows the simulation results for $S_{12}$ versus $G$. Increasing $G$ from $0.2 \mathrm{~mm}$ to $0.5 \mathrm{~mm}$ lead to increasing resonance frequency and rejection bandwidth. Also, in case of $G$ variation, $Q$-factor is not change considerably. All other parameters are set as in other cases to $B=1 \mathrm{~mm}, A=D=0.2 \mathrm{~mm}$, $H=3 \mathrm{~mm}$ and $C=14.2 \mathrm{~mm}$.

The simulated forward transmission versus $H$ parameter varies from $2.5 \mathrm{~mm}$ to $4 \mathrm{~mm}$ with $0.5 \mathrm{~mm}$ steps are shown in Fig. 6. In this case other parameters are set to $B=1 \mathrm{~mm}$, $A=D=G=0.2 \mathrm{~mm}$ and $C=14.2 \mathrm{~mm}$. It can be seen that by increasing $H$, both parameters, rejection bandwidth and the resonance frequency, are decreased, whereas $Q$-factor is not changed significantly.

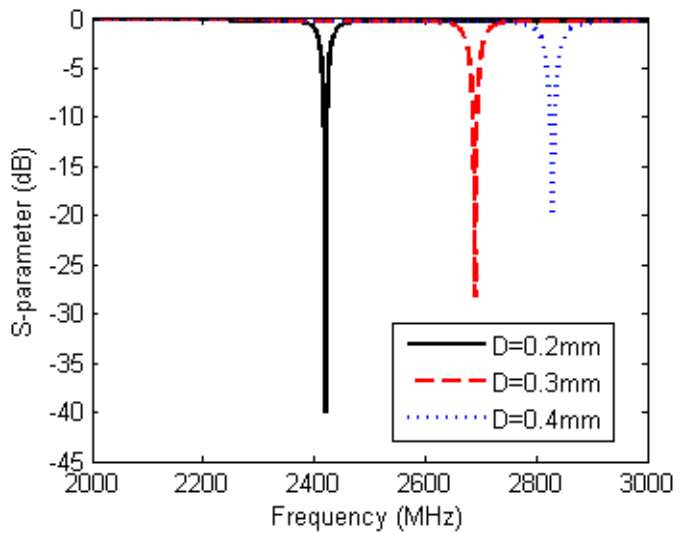

Fig. 3. Simulated $S_{12}$ of the DGS filter versus frequency for various $D$.

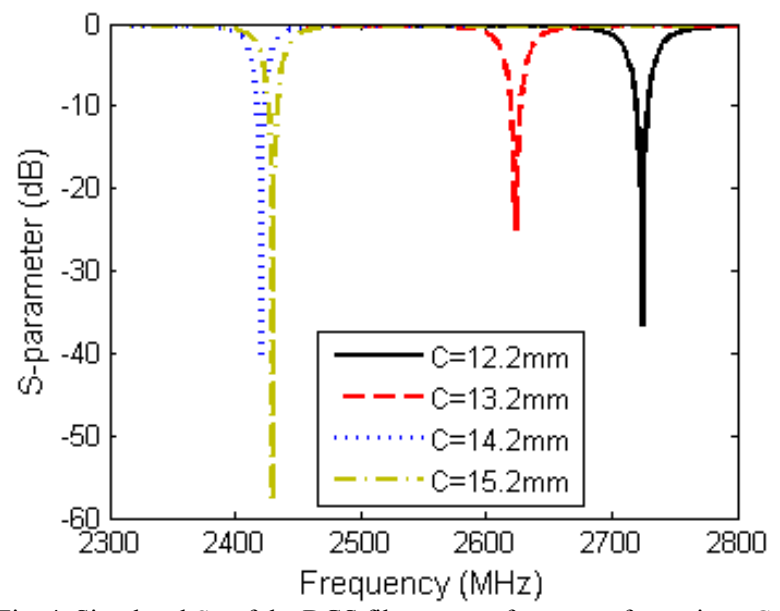

Fig. 4. Simulated $S_{12}$ of the DGS filter versus frequency for various $C$.

\section{EXPERIMENTAL RESULTS OF DGS FILTER}

To verify the obtained numerical results, the $C$-shaped DGS filter was fabricated using PC-board RF-35. The optimized value of the DGS parameters, as mentioned earlier, are $A=D=G=0.2 \mathrm{~mm}, B=1 \mathrm{~mm}, H=3 \mathrm{~mm}, C=14.2 \mathrm{~mm}$, and PC-boars size is set to $30 \times 40 \mathrm{~mm}^{2}$. The top and bottom views of the fabricated band stop filter are shown in Fig. 7. Return loss and forward transmission of the designed and implemented filter was measured using HP8510 network analyzer.

In Fig. 8, measured $S_{11}$ and $S_{12}$ are presented at two different frequency ranges. It can be seen that proposed filter exhibits a very low insertion loss at the pass-band and also provides a sharp frequency response in transition band. 
Rejection bandwidth is $43 \mathrm{MHz}$, so the measured $Q$-factor is 57.5. The suppression for the resonance frequency, 2.483 $\mathrm{GHz}$, is $24 \mathrm{~dB}$. The measured transfer characteristic of the proposed fabricated $C$-shaped band-reject filter shows steep band-rejection property providing a very high $Q$-factor.

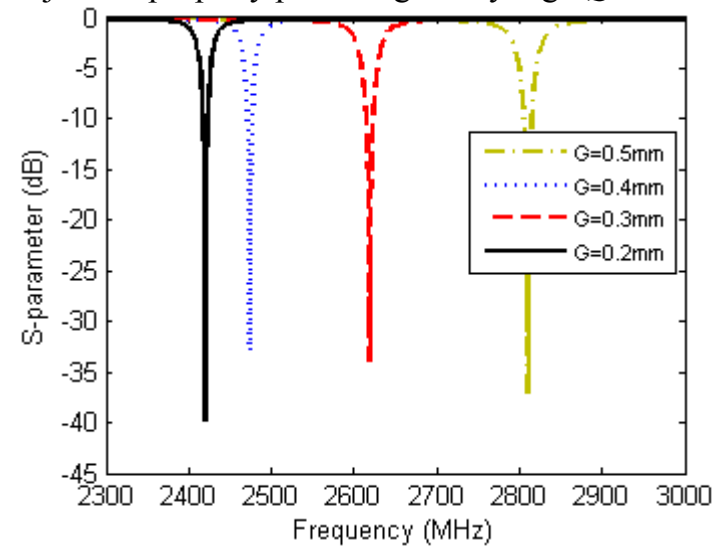

Fig. 5. Simulated $S_{12}$ of the DGS filter versus frequency for various $G$.

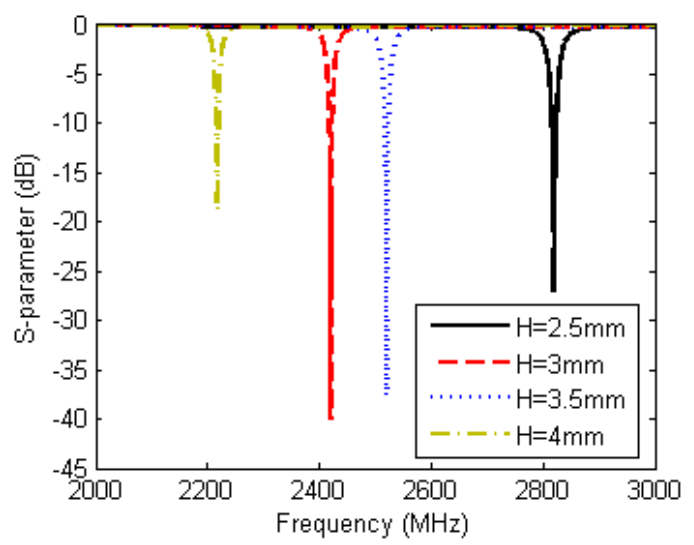

Fig. 6. Simulated $S_{12}$ of DGS filter versus frequency for various $H$.

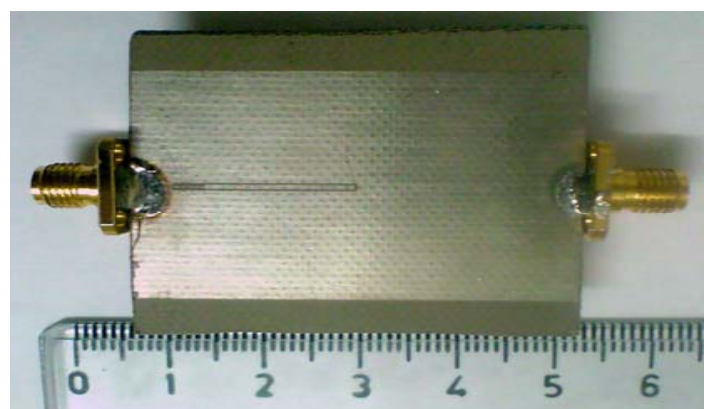

(a)

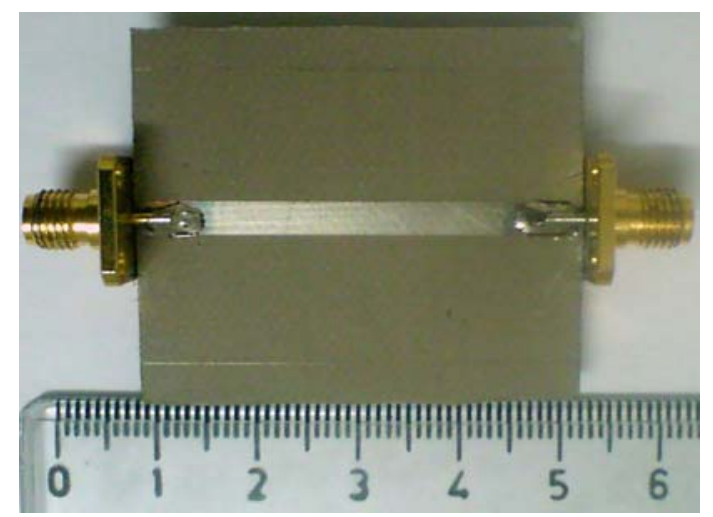

(b)

Fig. 7. Fabricated $C$-shaped DGS band-reject filter: a) bottom view, b) top view.

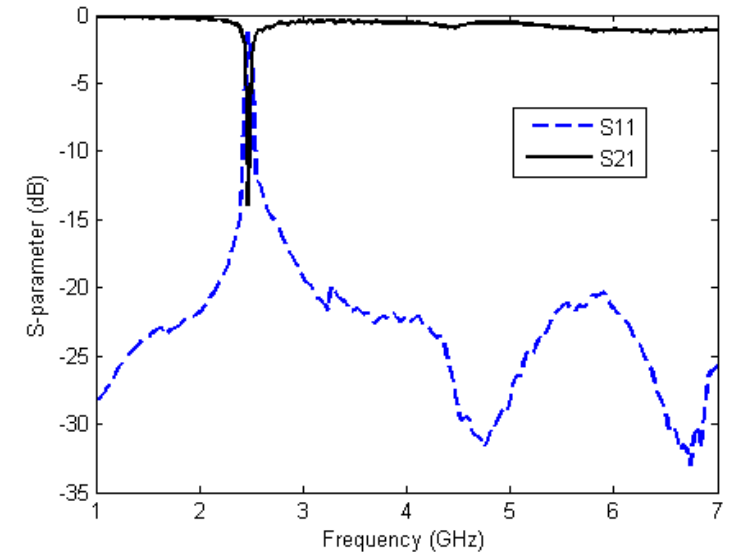

(a)

(a)

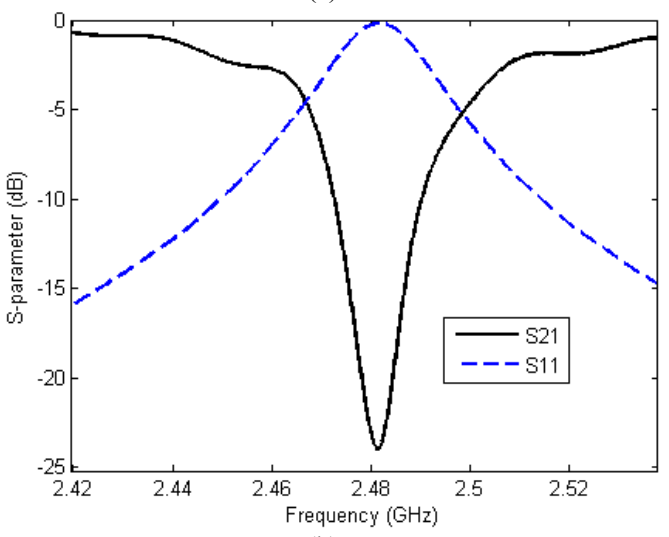

(b)

Fig. 8. Measured $S$-parameters of the $C$-shaped DGS filter: a) form $1 \mathrm{GHz}$ to $7 \mathrm{GHz}, \mathrm{b}$ ) around the resonance frequency.

\section{CONCLUSION}

In this paper, a new novel $C$-shaped DGS unit with improved $Q$-factor of the band-rejection characteristics has been introduced. A parametric study of the effects of $C$-shaped DGS parameter dimensions on the frequency performance of the filter were numerically investigated by HFSS. The best values of DGS dimensions were determined for lower bandwidth at the resonance frequency around 2.45 GHz. The DGS filter was fabricated and tested using a network analyzer. The measured results show a good agreement with simulated ones. It provides a parallel resonance frequency of $2.483 \mathrm{GHz}$ with $24 \mathrm{~dB}$ suppression. The measured rejection bandwidth is $43 \mathrm{MHz}$ with a $Q$-factor of 57.5 and with attenuation of $24 \mathrm{~dB}$ at resonant frequency. The proposed filter may have wide applications in the design of microwave systems and antenna arrays, where small size and high $Q$-factor is needed.

\section{ACKNOWLEDGMENT}

The authors would like to appreciate Iran Telecommunication Research Center (ITRC) for their financial support.

\section{REFERENCES}

[1] J. S. Park and M. S. Jung, "A novel defected ground structure for an active device mounting an application to a microwave oscillator," IEEE Microw. Wireless Compon. Lett., vol. 14, no. 5, pp. 198-200, May 2004. 
[2] D. J. Woo and T. K. Lee, "Suppression of harmonics in Wilkinson power divider by using asymmetric spiral DGS," in Proc. of 34 th Eur. Microwave Conf. Dig., vol. 1, Oct. 2004, pp. 467-470.

[3] C. S. Kim, J. S. Park, D. Ahn, and J. B. Lim, "A novel 1-D periodic defected ground structure for planar circuits," IEEE Microw. Guided Wave Lett., vol. 10, no. 4, pp. 131-133, Apr. 2000.

[4] D. J. Woo, T. K. Lee, J. W. Lee, C. S. Pyo, and W. K. Choi, "Novel U-slot and V-slot DGSs for bandstop filter with improved Q factor," IEEE Trans. Microw. Theory Tech., vol. 54, no. 6, pp. 2840-2847, Jun. 2006.

[5] J. S. Park, J. S. Yun, and D. Ahn, "A design of the novel coupled-line bandpass filter using defected ground structure with wide stopband performance," IEEE Trans. Microw. Theory Tech., vol. 50, no. 9, pp. 2037-2043, Sep. 2002.

[6] J. S. Lim, Y. C. Jeong, D. Ahn, and S.W. Nam, "A technique reducing the size of microwave amplifiers using spiral-shaped defected ground structure,” J. Korea Electromagn. Eng, vol. 14, no. 9, pp. 904-911, Sep. 2003.

[7] D. Ahn, J. S. Park, C. S. Kim, J. N. Kim, Y. Qian, and T. Itoh, “A design of the low-pass filter using the novel microstrip defected ground structure," IEEE Trans. Microw. Theory Tech., vol. 49, no. 1, pp. 86-93, Jan. 2001.

[8] K. H. Yi and B. K. Kang, "Modified Wilkinson power divider for $n$th harmonic suppression," IEEE Microw. Wireless Compon. Lett., vol. 13, no. 5, pp. 178-180, May 2003.

[9] D. J. Woo and T. K. Lee, "Suppression of harmonics in Wilkinson power divider using dual-band rejection by asymmetric DGS," IEEE Trans. Microw. Theory Tech, vol. 53, no. 6, pp. 2139-2144, Jun. 2005.

[10] J. S. Lim, C. S. Kim, D. Ahn, and Y. C. Jeong, "Design of low-pass filter using defected ground structure," IEEE Trans. Microw. Theory Tech, vol. 53, no. 8, pp. 2539-2545, Aug. 2005.

[11] S. Y. Huang and Y. H. Lee, "A compact E-shaped patterned ground structure and its applications to tunable bandstop resonator," IEEE Transactions on Microwave Theory and Techniques, vol. 57, no. 3, pp. 657-666, Mar., 2009.

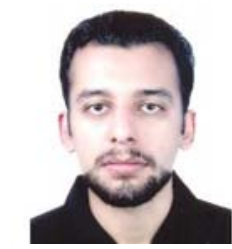

DGS structures.

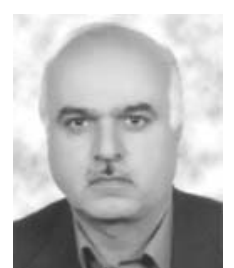

Mohammad $\mathbf{H}$. Neshati was born in Yazd, Iran. He received the B.S. degree from Isfahan University of Technology, Isfahan, Iran, in 1984, the M.S. degree from Amir Kabir University of Technology, Tehran, Iran, in 1988, and the Ph.D. degree from the University of Manchester Institute of Science and Technology (UMIST), Manchester, U.K., in 2000, all in electrical engineering. In 1889, he joined the faculty of engineering at the University of Sistan and Baluchistan, Zahedan, Iran, where he taught electromagnetics, field theory, microwave circuits and conducted research in the development of microwave antennas especially on dielectric resonator antennas (DRAs). He recently joined the Electrical Engineering Department, Ferdowsi University of Mashhad, Mashhad, Iran, as an Assistant Professor here he conducts research in EM theory, antennas and microwave circuits. 